\title{
CURVATURE IN HAMILTONIAN MECHANICS AND THE EINSTEIN-MAXWELL-DILATON ACTION
}

\author{
S. G. RAJEEV
}

\begin{abstract}
Riemannian geometry is a particular case of Hamiltonian mechanics: the orbits of the hamiltonian $H=\frac{1}{2} g^{i j} p_{i} p_{j}$ are the geodesics. Given a symplectic manifold $(\Gamma, \omega)$, a hamiltonian $H: \Gamma \rightarrow \mathbb{R}$ and a Lagrangian submanifold $M \subset \Gamma$ we find a generalization of the notion of curvature. The particular case $H=\frac{1}{2} g^{i j}\left[p_{i}-A_{i}\right]\left[p_{j}-A_{j}\right]+\phi$ of a particle moving in a gravitational, electromagnetic and scalar fields is studied in more detail. The integral of the generalized Ricci tensor w.r.t. the Boltzmann weight reduces to the action principle $\int\left[R+\frac{1}{4} F_{i k} F_{j l} g^{k l} g^{i j}-g^{i j} \partial_{i} \phi \partial_{j} \phi\right] e^{-\phi} \sqrt{g} d^{n} q$ for the scalar, vector and tensor fields.
\end{abstract}

\section{INTRODUCTION}

The theory of geodesics on a Riemannian manifold is a particular case of Hamiltonian mechanics: they are simply the solutions of Hamilton's equations for $H=$ $\frac{1}{2} g^{i j} p_{i} p_{j}$. Is there a generalization of Riemannian geometry corresponding to more general hamiltonians? The three ideas we would like to generalize are those of distance, volume and curvature.

The volume is the easiest to generalize: the Boltzmann weight gives a natural measure of integration in the phase space. Integrating out the momenta gives the generalization of the Riemannian volume element in configuration space (as long as $\int e^{-H} d^{n} p$ converges).

Recall that the "reduced action" (a.k.a "eikonal") $\sigma_{E}\left(Q, Q^{\prime}\right)=\int_{Q^{\prime}}^{Q} p_{i}(E, q) d q^{i}$ of the trajectory of given energy $E$ connecting two points is a good candidate for distance; although in the most general case it is not positive or symmetric, let alone satisfy the triangle inequality. If the Hamiltonian is an even function of momenta $H(q, p)=H(q,-p)$ we have time reversal invariance and the symmetry $\sigma_{E}\left(Q, Q^{\prime}\right)=\sigma_{E}\left(Q^{\prime}, Q\right)$ follows. If in addition $H(q, p)$ is a convex function of the momenta, $\sigma_{E}\left(Q, Q^{\prime}\right)$ satisfies the triangle inequality. In particular, for familiar mechanical systems it reduces to the Jacobi-Maupertuis metric.

The most subtle notion to generalize is curvature. We will use the second variation of the action to find a quantity $\mathcal{R}_{i j}(q, p)$ which transforms as a symmetric tensor under co-ordinate transformations in configuration space and which reduces to the Riemann tensor in Riemannian geometry: $\mathcal{R}_{i j}(q, p)=-g_{i m}(q) p^{k} p^{l} R_{k l j}^{m}(q)$ where two of the indices of the Riemann tensor are contracted by momentum.

An explicit formula for curvature in terms of derivatives of the hamiltonian (up to fourth order) will be given. Its trace is the generalization of the Ricci form; integrating over momentum gives the analogue of the Ricci scalar density. We will show that in the case of most physical interest,

Date: Jan 152017. 


$$
H(q, p)=\frac{1}{2} g^{i j}(q)\left[p_{i}-A_{i}(q)\right]\left[p_{j}-A_{j}(q)\right]+\phi(q)
$$

this Ricci scalar density is a natural unified action for gravity coupled to electromagnetic and dilaton fields. Usually such actions arise from much more complicated theories with many unwanted fields (Kaluza-Klein, String theory etc.).

To further strengthen our claim of having found the correct generalization, we will show that in some simple but rather subtle cases (Lagrange points, Penning trap) the positivity of curvature is sufficient for stability; while negative curvature implies instability.

The simple harmonic oscillator has constant positive curvature; it also has finite diameter for the allowed subset of states of the configuration space for a given a energy. Its phase space has finite volume w.r.t. to the Boltzmann measure. Thus the simple harmonic oscillator is the mechanical analogue of the sphere $\mathbb{S}^{2}$.

In Riemannian geometry, Myer's theorem says that the diameter is finite when the curvature is bounded below by a positive constant. Under the same condition, the Lichnerowicz theorem says that the spectrum of the Laplacian has a gap (the first non-zero eigenvalue is bounded below by a constant). Perhaps there are generalizations to mechanical systems.

Let us spell out these ideas in some more detail before delving into calculations.

1.1. Distance. Even in Riemannian geometry, the natural way to measure distance between two points $Q^{\prime}, Q$ is to find the minimum over piece-wise differentiable curves of the action

$$
S=\frac{1}{2} \int_{0}^{T} g_{i j} \dot{q}^{i} \dot{q}^{j} d t, \quad q(0)=Q^{\prime}, \quad q(T)=Q
$$

rather than the arc-length

$$
l=\frac{1}{2} \int_{0}^{T} \sqrt{g_{i j} \dot{q}^{i} \dot{q}^{j}} d t
$$

The square root makes the arc-length tricky to differentiate as a function of the curve . (Just as $|x|$ is not differentiable, unlike $x^{2}$ ). In the jargon of high energy physics, the re-parametrization invariance of $l$ is a "gauge invariance" that needs to be fixed; $S$ is a "gauge-fixed" version of $l . S$ is called energy in many mathematics textbooks 1], but the proper mechanical analogue is action.

Let $s_{T}\left(Q, Q^{\prime}\right)$ be the value of $S$ on the minimizing curve. It is related to the Riemannian distance $d\left(Q, Q^{\prime}\right)$ (i.e., the minimum of the arc-length) through

$$
s_{T}\left(Q, Q^{\prime}\right)=\frac{d^{2}\left(Q, Q^{\prime}\right)}{2 T} .
$$

It satisfies the Hamilton-Jacobi equation

$$
\frac{1}{2} g^{i j} \partial_{Q^{i}} s_{T} \partial_{Q^{j}} s_{T}+\frac{\partial s_{T}}{\partial T}=0 .
$$

Its Legendre transform

$$
\sigma_{E}\left(Q, Q^{\prime}\right)=\min _{T}\left[E T+s_{T}\left(Q, Q^{\prime}\right)\right], \quad \sigma_{E}\left(Q, Q^{\prime}\right)=\sqrt{2 E} d\left(Q, Q^{\prime}\right)
$$


satisfies the stationary Hamilton-Jacobi equation (the eikonal equation in the language of optics)

$$
\frac{1}{2} g^{i j} \partial_{Q^{i}} \sigma_{E} \partial_{Q^{j}} \sigma_{E}=E .
$$

Because $T, E$ only appear as overall factors in these formulas, it is customary in geometry to choose units where they are set to constant values $\left(T=\frac{1}{2}=E\right)$; this corresponds to choosing a parametrization where the velocity of the geodesic is unity.

For a more general mechanical system the action is still some integral over the curve

$$
S=\int L(q, \dot{q}) d t
$$

but it may not be a purely quadratic function of the velocities. If we drop the condition that $L(q, \dot{q})$ be quadratic in $\dot{q}$, but insist that it is homogenous

$$
L(q, \lambda \dot{q})=\lambda^{r} L(q, \dot{q}), \quad \lambda, r>0
$$

we get the well-studied case of Finsler geometry $[2,3]$. As Chern points out, this is the case originally studied by Riemann in his Habilitation; later, the name Riemannian geometry came to be associated to the restricted case of a quadratic form. Finsler geometry is so natural that Chern says 2

"Finsler geometry is not a generalization of Riemannian geometry.

It is better described as Riemannian geometry without the quadratic restriction".

The typical hamiltonian of a mechanical system is a quadratic, but not homogenous function of momenta

$$
H(q, p)=\frac{1}{2} g^{i j}\left[p_{i}-A_{i}(q)\right]\left[p_{j}-A_{j}(q)\right]+\phi(q)
$$

corresponding to a Lagrangian

$$
L(q, \dot{q})=\frac{1}{2} g_{i j} \dot{q}^{i} \dot{q}^{j}+A_{i} \dot{q}^{i}-\phi(q)
$$

This describes a particle moving under the influence of gravitational, electromagnetic and scalar forces. It is natural look for a geometry associated to such (or even more general) hamiltonians. To paraphrase Chern, we seek

"Riemannian Geometry Without the Homogenous Restriction".

Such ideas go back to Hamilton [4] himself, as noted by Klimes [5].

1.2. Hamiltonian Mechanics. Let us recall some basic facts of mechanics. By Darboux's theorem [6] every symplectic manifold $(\Gamma, \omega)$ can be covered by co-ordinate charts such that in each chart the symplectic form has constant coefficients:

$$
\omega=d p_{i} d q^{i} .
$$

These co-ordinates satisfy the canonical Poisson brackets

$$
\left\{q^{i}, q^{j}\right\}=0=\left\{p_{i}, p_{j}\right\}, \quad\left\{p_{i}, q^{j}\right\}=\delta_{i}^{j}
$$


A hamiltonian $H: \Gamma \rightarrow \mathbb{R}$ determines a family of curves that pass through each point of $M$, satisfying Hamilton's equations

$$
\dot{q}^{i}=H^{i}, \quad \dot{p}_{i}=-H_{i}
$$

where

$$
\dot{q}^{i}=\frac{d q^{i}}{d t}, \quad H^{i}=\frac{\partial H}{\partial p_{i}}, \quad H_{i}=\frac{\partial H}{\partial q^{i}} .
$$

These curves are the extrema of the action

$$
S=\int\left[p_{i} \dot{q}^{i}-H\right] d t
$$

If we are also given a Lagrangian sub-manifold $M \subset \Gamma$, locally $\Gamma$ can be identified $[6]$ with the co-tangent bundle $T^{*} M$. That is, there is a neighborhood in which $M$ is determined by $p_{i}=0$ so that $q^{i}$ are co-ordinates on it. An example is the case where $M$ is the configuration space of a physical system.

Given a pair of points that are close enough and a time $T$, there is a solution to Hamilton's equations with the boundary conditions

$$
q^{i}(0)=Q^{i \prime}, \quad q^{i}(T)=Q^{i}
$$

The action of this solution $s_{T}\left(Q, Q^{\prime}\right)$ has a Legendre transform

$$
\sigma_{E}\left(Q, Q^{\prime}\right)=\min _{T}\left[E T+s_{T}\left(Q, Q^{\prime}\right)\right]
$$

They satisfy the time dependent

$$
H\left(Q, \partial_{Q} s_{T}\right)+\frac{\partial s_{T}}{\partial T}=0
$$

and stationary

$$
H\left(Q, \partial_{Q} \sigma_{E}\right)=E
$$

versions of the Hamilton-Jacobi equation. Thus $\sigma_{E}\left(Q, Q^{\prime}\right)$ can be thought of as a generalization of the Riemannian distance function. We will adopt terminology from optics and call $\sigma_{E}\left(Q, Q^{\prime}\right)$ the eikonal.

But there are important differences; it may not be a homogenous function of $E$ unless the hamiltonian happens to have some sort of scale symmetry. In general, it is not symmetric:

$$
\sigma_{E}\left(Q, Q^{\prime}\right) \neq \sigma_{E}\left(Q^{\prime}, Q\right)
$$

An example is a particle moving in a magnetic field (there is an explicit calculation below). Thus, we will not be able to define a metric (in the sense of topology) on the Lagrangian manifold $M$ (configuration space) using $\sigma_{E}$. But that should not bother physicists too much: we already gave that up when we allowed $g_{i j}$ to have Lorentzian signature in relativistic mechanics. 
1.2.1. Time Reversal Invariant systems. If the hamiltonian is time reversal invariant

$$
H(q, p)=H(q,-p)
$$

the eikonal will be a symmetric function

$$
\sigma_{E}\left(Q, Q^{\prime}\right)=\sigma_{E}\left(Q^{\prime}, Q\right)
$$

An example is the case of a particle moving in a potential but with no magnetic field:

$$
H(q, p)=\frac{1}{2} g^{i j} p_{i} p_{j}+V(q)
$$

In this example the stationary Hamilton-Jacobi equation can be rewritten as

$$
\frac{1}{2[E-V(Q)]} g^{i j} \partial_{Q^{i}} \sigma_{E} \partial_{Q^{j}} \sigma_{E}=1
$$

which is just the eikonal equation for the Jacobi-Maupertius metric

$$
\hat{g}_{i j}(q)=[E-V(q)] g_{i j}(q) .
$$

The trajectories must lie in the region where $V(q)<E$; they are geodesics of the Jacobi-Maupertius metric on the manifold whose boundary consist of turning points where $E=V(q)$. In particular, $\sigma_{E}\left(Q, Q^{\prime}\right)$ satisfies the triangle inequality and is a metric (in the sense of topology) .

This example suggests that in the case of time reversal invariant systems for which $H(q, p)$ is a convex function of momenta, (i.e., $H^{i j}(q, p) \equiv \frac{\partial^{2} H}{\partial p_{i} \partial p_{j}}$ is a positive matrix), the eikonal $\sigma_{E}\left(Q, Q^{\prime}\right)$ is a metric in the sense of topology on some subset $M_{E} \subset M$ of allowed configurations. It would be interesting to have a rigorous mathematical proof of this.

Some Remarks:

- Is there a version of Myer's theorem[1]? That is, given that the Ricci curvature(defined below) is bounded below $\mathcal{R} \geq \omega^{2}>0$, does it follow that $\sigma_{E}\left(Q, Q^{\prime}\right) \leq \pi \frac{E}{\omega}$ ? We will see some elementary examples that suggest that this is true. Is the fundamental group of $M_{E}$ finite?

- In the other direction, does $-\mathcal{R} \geq \omega^{2}>0$ and boundedness of $\sigma_{E}$ imply that $M_{E}$ has infinite fundamental group? This could have applications to ergodicity.

- When we pass to the quantum theory, $\sigma_{E}$ becomes the phase of the wave function and the HJ equation becomes the Schrodinger equation. In the toy model where $M$ is one-dimensional, it is possible to find a quantum theory of gravity based on this interpretation 8 . Perhaps it is of interest to see how much of that generalizes to higher dimensions.

1.3. Volume. Given a hamiltonian $H: \Gamma \rightarrow \mathbb{R}$, there is a natural measure of integration on phase space (motivated by Thermodynamics), the Boltzmann weight(Of course, $n=\frac{1}{2} \operatorname{dim} \Gamma$.)

$$
d \mu_{H}=e^{-H} \frac{d^{n} p d^{n} q}{[2 \pi]^{\frac{n}{2}}} .
$$


It is normalized to agree, in that special case, with the Riemannian volume $\sqrt{g} d^{n} q$ on the configuration space (after integrating out the momentum directions). Also, we have chosen units in which the temperature is equal to one.

1.4. Curvature. As noted earlier, there is no obstruction to choosing local coordinates in which the symplectic form has constant components. So, unlike a Riemannian metric, a symplectic form does not uniquely determine a connection or curvature. There are many torsion-less connections, that preserve the symplectic form; there is no local obstruction to choosing the curvature to be zero. There could be global obstructions however. It is possible to choose a connection on a symplectic manifold by a variational principle 9 . This is useful in deformation quantization. None of this has any dependence on the hamiltonian.

Instead, we want to construct a curvature from the Hamiltonian that measures the response of a mechanical system to small perturbations. But a hamiltonian $H$, curvature. For example, there is a neighborhood of every minimum of $H$ in which the Hamiltonian can be brought to the Birkhoff Normal Form[7]. Assuming that the natural frequencies of small oscillations at the minimum are not rationally related (which is the generic case) there is a canonical transformation that brings $H$ to the quadratic form

$$
H(q, p)=\frac{1}{2} \sum_{k}\left[p_{k}^{2}+\omega_{k}^{2} q_{k}^{2}\right]+\cdots
$$

up to any desired order $\geq 3$ in $p_{k}, q_{k}$.

In Riemannian geometry, the infinitesimal deviation of geodesics (which is determined by the second variation of the action) determines curvature. It would be useful to have a generalization of curvature to more general mechanical systems. For example, negative curvature could be an indication of dynamical instability [10, 11].

Given a symplectic manifold $\Gamma$, a hamiltonian $H: \Gamma \rightarrow \mathbb{R}$ and a Lagrangian sub-manifold $M \subset \Gamma$ we will construct a notion of curvature. The trick is to again consider the second variation of the action. We will be able to write it as

$$
\mathcal{S}_{1}=\int\left[\frac{1}{2} G_{i j}(q, p) \stackrel{\circ}{\dot{ }^{i} \circ j} \dot{\xi}-\frac{1}{2} \xi^{i} \xi^{j} \mathcal{R}_{i j}(q, p)\right] d t
$$

Here $\xi$ is the infinitesimal variation of the orbit, thought of as a curve in $M$.

Also, $\stackrel{\circ}{\xi}^{i}$ is a covariant derivative of $\xi$ along the orbit. (The explicit formula is given later). We do not attempt to define a covariant derivative (connection, parallel transport etc.) along an arbitrary direction.

$G_{i j}(q, p)$ is the inverse matrix of the second derivative of the hamiltonian w.r.t. momentum

$$
H^{i k} G_{k j}=\delta_{j}^{i}, \quad H^{i j}=\frac{\partial H}{\partial p_{i} \partial p_{j}} .
$$

We will require that this second derivative $H^{i j}$ of the hamiltonian be a positive matrix, so that the inverse exists. (It will be clear in most cases how to do a "Wick Rotation" to the case (e.g., of Lorentzian signature) when $H^{i j}$ is only invertible and not positive.) That is, we require that $H(q, p)$ is a convex function of momenta. We will prove that the quantities $G_{i j}(q, p), \mathcal{R}_{i j}(q, p)$ transform as symmetric tensors under co-ordinate transformations $q^{i} \rightarrow \tilde{q}^{i}(q)$. They are not in general homogenous 
functions of $p$. An explicit expression for the curvature tensor $\mathcal{R}_{i j}(q, p)$ in terms of derivatives (up to fourth order) of $H$ will be given.

There is also an analogue of the Ricci tensor

$$
\mathcal{R}(q, p)=H^{i j} \mathcal{R}_{i j}(q, p)
$$

The generalization of the Ricci scalar-density is its average over momentum:

$$
\mathfrak{R}(q)=\int \mathcal{R}(q, p) e^{-H(q, p)} \frac{d^{n} p}{[2 \pi]^{\frac{n}{2}}} .
$$

1.4.1. Riemannian Geometry. In the particular case of Riemannian geometry

$$
H(q, p)=\frac{1}{2} g^{i j}(q) p_{i} p_{j}
$$

they reduce to the Riemann tensor $R_{k l j}^{m}$, Ricci tensor $R_{i j}$ and the Ricci scalardensity as follows:

$$
\mathcal{R}_{i j}(q, p)=-g_{i m} H^{k} H^{l} R_{k l j}^{m}(q), \quad \mathcal{R}(q, p)=H^{k} H^{l} R_{k l}(q), \quad \mathfrak{R}(q)=\sqrt{g} R(q)
$$

where

$$
H^{k}=g^{k m} p_{m} .
$$

Thus $\mathscr{R}$ is the Einstein-Hilbert Lagrangian density for GR (with Euclidean signature).

1.4.2. Adding a Magnetic Field. The hamiltonian

$$
H=\frac{1}{2} g^{k l}\left[p_{k}-A_{k}\right]\left[p_{l}-A_{l}\right]
$$

leads to

$$
\begin{gathered}
\mathcal{R}_{i j}=-g_{i m} H^{k} H^{l} R_{k l j}^{m}+\frac{1}{4} F_{i k} F_{j l} g^{k l}+\frac{1}{2} H^{k}\left\{\partial_{j} F_{k i}+\partial_{i} F_{k j}\right\} \\
\mathcal{R}=H^{k} H^{l} R_{k l}+\frac{1}{4} F_{i k} F_{j l} g^{k l} g^{i j}+H^{k} g^{i j} \partial_{i} F_{k j} \\
\mathscr{R}=\left[R+\frac{1}{4} F_{i k} F_{j l} g^{k l} g^{i j}\right] \sqrt{g}
\end{gathered}
$$

Thus $\mathscr{R}$ is exactly the Lagrangian density for Einstein-Maxwell theory. We get the correct "unified" variational principle in a natural geometric theory without having to assume extra dimensions (as in Kaluza-Klein theory). 
1.4.3. Adding a Scalar Field. If we add also a scalar potential

$$
\begin{gathered}
H=\frac{1}{2} g^{k l}\left[p_{k}-A_{k}\right]\left[p_{l}-A_{l}\right]+\phi \\
\mathcal{R}_{i j}=-g_{i m} H^{k} H^{l} R_{k l j}^{m}+\frac{1}{4} F_{i k} F_{j l} g^{k l}+\frac{1}{2} H^{k}\left\{\partial_{j} F_{k i}+\partial_{i} F_{k j}\right\}+\partial_{i} \partial_{j} \phi
\end{gathered}
$$

In particular, the curvature of a non-relativistic particle with potential energy $\phi$ is simply its Hessian $\partial_{i} \partial_{j} \phi$. The harmonic oscillator has constant positive curvature. The inverted harmonic oscillator (which has an unstable equilibrium point) has constant negative curvature. The Ricci curvature is

$$
\mathcal{R}=H^{k} H^{l} R_{k l}+\frac{1}{4} F_{i k} F_{j l} g^{k l} g^{i j}+H^{k} g^{i j} \partial_{i} F_{k j}+\Delta \phi
$$

Again for a a non-relativistic particle the Ricci curvature is the Laplacian of the potential.

The generalization of the Ricci scalar density in this case

$$
\Re=\left[R+\frac{1}{4} F_{i k} F_{j l} g^{k l} g^{i j}+\Delta \phi\right] e^{-\phi} \sqrt{g}
$$

Similar expression also arise as effective Lagrangian densities in string theory and in Kaluza-Klein theories; the scalar $\phi$ is the dilaton in that context ${ }^{1}$

If we make the field redefinition

$$
\tilde{g}_{i j}=e^{2 \alpha \phi} g_{i j}
$$

and choose

$$
\alpha=-\frac{1}{n-2}
$$

the scalar curvature density can be brought to the more conventional form (dropping a total derivative)

$$
\mathfrak{R}=\sqrt{\tilde{g}} \tilde{R}+\frac{1}{4} F_{i k} F_{j l} \tilde{g}^{k l} \tilde{g}^{i j} \sqrt{\tilde{g}} e^{-\frac{2}{n-2} \phi}+\frac{2 n-1}{n-2} \sqrt{\tilde{g}} \tilde{g}^{i j} \partial_{i} \phi \partial_{j} \phi
$$

This action describes a scalar and a photon minimally coupled to the gravitational field, with an additional non-minimal coupling of the scalar to the photon. The parametrization of the original Hamiltonian is, for reference,

$$
H=\frac{1}{2} e^{-\frac{2}{n-2} \phi} \tilde{g}^{i j}\left[p_{i}-A_{i}\right]\left[p_{j}-A_{j}\right]+\phi .
$$

More specific examples are given later (Section 6). We now turn to the explicit calculations to establish these facts.

\footnotetext{
${ }^{1}$ I thank Sumit Das for clarifying this point.
} 


\section{Co-ORDinAte TRANSFORMATIONS}

Our considerations are local, best described in old fashioned co-ordinate notation. It is important to know how quantities transform under co-ordinate transformations and to identify tensorial quantities, which transform homogeneously.

Let us begin with Hamilton's equations themselves. The configuration space $M$ has co-ordinates $q^{i}$, which determine a canonical co-ordinate system on $\Gamma$ with conjugate variables $p_{i}$. We can transform to any new set of co-ordinates $\tilde{q}^{i}$ which are smooth functions of $q^{i}$ such that the inverse transformation is smooth as well. The momenta $\tilde{p}_{i}$ conjugate to $\tilde{q}^{i}$ are given by the transformation law of covariant vectors fields (components of a 1 -form)

$$
\tilde{p}_{i}=\frac{\partial q^{j}}{\partial \tilde{q}^{i}} p_{j}
$$

It follows that $\dot{q}^{i}$ and $H^{i}=\frac{\partial H}{\partial p_{i}}$ transform as the components of a contra-variant vector field.

$$
H^{j}=\frac{\partial \tilde{q}^{j}}{\partial q^{i}} H^{i}
$$

But $\dot{p}_{i}$ and $H_{i}=\frac{\partial H}{\partial q^{i}}$ do not transform homogeneously. Instead,

$$
\tilde{H}_{j}=H_{b} \frac{\partial q^{b}}{\partial \tilde{q}^{j}}+H^{a} \tilde{p}_{k} \frac{\partial^{2} \tilde{q}^{k}}{\partial q^{c} \partial q^{a}} \frac{\partial q^{c}}{\partial \tilde{q}^{j}}
$$

We can see this by rewriting

$$
d H=H_{i} d q^{i}+H^{i} d p_{i}
$$

in the new canonical co-ordinate system:

$$
\begin{gathered}
d H=H_{i} \frac{\partial q^{i}}{\partial \tilde{q}^{a}} d \tilde{q}^{a}+H^{i} d\left\{\tilde{p}_{a} \frac{\partial \tilde{q}^{a}}{\partial q^{i}}\right\} \\
=H_{i} \frac{\partial q^{i}}{\partial \tilde{q}^{a}} d \tilde{q}^{a}+H^{i} \frac{\partial \tilde{q}^{a}}{\partial q^{i}} d \tilde{p}_{a}+H^{i} \tilde{p}_{a} \frac{\partial^{2} \tilde{q}^{a}}{\partial q^{j} \partial q^{i}} d q^{j}
\end{gathered}
$$

By collecting the coefficients of $d \tilde{p}_{j}, d \tilde{q}^{j}$ we get the above transformation laws for $\tilde{H}^{j}, \tilde{H}_{j}$. It will be convenient to denote the various derivatives of the hamiltonian by

$$
H_{j_{1} \cdots j_{s}}^{i_{1} \cdots i_{r}}=\frac{\partial^{r+s} H}{\partial p_{i_{1}} \cdots \partial p_{i_{r}} \partial q^{j_{1}} \cdots \partial q^{j_{s}}}
$$

That is, the upper indices correspond to differentiation with respect to $p_{i}$ and the lower indices to $q^{i}$. By extension of the above argument, we see that $H^{i_{1} \cdots i_{r}}$ transform as the components of a symmetric tensor under canonical co-ordinate transformations; but that the mixed derivatives $H_{j_{1} \cdots j_{s}}^{i_{1} \cdots i_{r}}=\frac{\partial^{r+s} H}{\partial p_{i_{1}} \cdots \partial p_{i_{r}} \partial q^{j_{1}} \cdots \partial q^{j_{s}}}$ with $s>0$ transform inhomogeneously.

We are assuming that the matrix $H^{i j}$ is positive; so it has an inverse $G_{j k}$ at every point $(q, p)$.

$$
H^{i j} G_{j k}=\delta_{k}^{i} .
$$


CURVATURE IN HAMILTONIAN MECHANICS AND THE EINSTEIN-MAXWELL-DILATON ACTIOIN

This $G_{i j}$ (which could depend on $p$ as well as $q$ ) is our analogue of the metric tensor; in particular it transforms covariant tensor. But we will not use $G_{i j}$ or $H^{i j}$ to raise or lower indices (except when we talk of the special case of Riemannian geometry).

Remark. The curvature computation makes sense if $H^{i j}$ as long as invertible, even if not positive (as in Lorentzian geometry). It would be interesting to generalize to the case where $H^{i j}$ are not invertible ("sub-Hamiltonian geometry"), analogous to sub-Riemannian geometry[12]. In fact, this paper arose out of my attempts to find a formula for curvature in sub-Riemannian geometry.

\section{The Second Variation}

Under the variation $q^{i} \mapsto q^{i}+\epsilon \xi^{i}, p_{i} \mapsto p_{i}+\epsilon \pi_{i}$ the change of the action $S=$ $\int\left[p_{i} \dot{q}^{i}-H\right] d t$ is, to second order,

$$
S_{\epsilon}=S+\epsilon \int\left[\pi_{i} \dot{q}^{i}+p_{i} \dot{\xi}^{i}-H_{i} \xi^{i}-H^{i} \pi_{i}\right] d t+\epsilon^{2} \int\left[\pi_{i} \dot{\xi}^{i}-\mathcal{H}\right] d t+\mathrm{O}\left(\epsilon^{3}\right)
$$

where

$$
\mathcal{H}=\frac{1}{2}\left[H_{i j} \xi^{i} \xi^{j}+2 H_{i}^{j} \xi^{i} \pi_{j}+H^{i j} \pi_{i} \pi_{j}\right]
$$

Requiring that the first order variation of $S$ be zero gives us Hamilton's equations. Given a solution of Hamilton's equations, the second variation ("Jacobi Functional")

$$
\mathcal{S}=\int\left[\pi_{i} \dot{\xi}^{i}-\mathcal{H}\right] d t
$$

can be thought of as the action of a mechanical system with quadratic (albeit time dependent) hamiltonian $\mathcal{H}$. It has an extremum when

$$
\dot{\xi}^{i}=H_{j}^{i} \xi^{j}+H^{i j} \pi_{j}, \quad \dot{\pi}_{i}=-H_{i j} \xi^{j}-H_{i}^{j} \pi_{j}
$$

The solutions of these equations are called "Jacobi fields". They describe the change of the orbit under infinitesimal perturbations of boundary conditions. Because $H^{i j}$ is invertible, we can eliminate $\pi_{i}$ in favor of $\dot{\xi}^{i}$

$$
\pi_{j}=G_{j k} \dot{\xi}^{k}-G_{j k} H_{l}^{k} \xi^{l}
$$

in the Jacobi functional to get a "Lagrangian" version of it:

$$
\mathcal{S}_{1}=\int\left[\frac{1}{2} G_{i j} \dot{\xi}^{i} \dot{\xi}^{j}-\dot{\xi}^{i} \xi^{j} G_{i k} H_{j}^{k}+\frac{1}{2} \xi^{i} \xi^{j}\left\{-H_{i j}+H_{i}^{k} H_{j}^{l} G_{k l}\right\}\right] d t
$$

We will mimic standard computations of Riemannian geometry 13 to regroup the integrand into tensorial terms. This will lead us to curvature.

Start with the identity

so that

$$
\dot{\xi}^{i} \xi^{j}=\frac{1}{2}\left[\dot{\xi}^{i} \xi^{j}-\dot{\xi}^{j} \xi^{i}\right]+\frac{1}{2} \frac{d}{d t}\left[\xi^{i} \xi^{j}\right]
$$

$$
-\dot{\xi}^{i} \xi^{j} G_{i k} H_{j}^{k}=-\frac{1}{2}\left[\dot{\xi}^{i} \xi^{j}-\dot{\xi}^{j} \xi^{i}\right] G_{i k} H_{j}^{k}-G_{i k} H_{j}^{k} \frac{1}{2} \frac{d}{d t}\left[\xi^{i} \xi^{j}\right]
$$


CURVATURE IN HAMILTONIAN MECHANICS AND THE EINSTEIN-MAXWELL-DILATON ACTION

$$
\begin{aligned}
& =\frac{1}{2} \dot{\xi}^{i} \xi^{j}\left[-G_{i k} H_{j}^{k}+G_{j k} H_{i}^{k}\right]+\frac{1}{2} \xi^{i} \xi^{j} \frac{d}{d t}\left[G_{i k} H_{j}^{k}\right]+\text { total derivative } \\
= & \frac{1}{2} \dot{\xi}^{i} G_{i k}\left[-H_{j}^{k}+H^{k m} G_{j l} H_{m}^{l}\right] \xi^{j}+\frac{1}{2} \xi^{i} \xi^{j} \frac{d}{d t}\left[G_{i k} H_{j}^{k}\right]+\text { total derivative }
\end{aligned}
$$

Imitating the calculation in Riemannian geometry, we also add another total derivative (this is the step that is not obvious):

allowing us to write

$$
\frac{d}{d t}\left[\frac{1}{4} \dot{G}_{i j} \xi^{i} \xi^{j}\right]=\frac{1}{2} \dot{G}_{i j} \dot{\xi}^{i} \xi^{j}+\frac{1}{4} \ddot{G}_{i j} \xi^{i} \xi^{j}
$$

$-\dot{\xi}^{i} \xi^{j} G_{i k} H_{j}^{k}=\frac{1}{2} \dot{\xi}^{i} G_{i k}\left[-H_{j}^{k}+H^{k m} G_{j l} H_{m}^{l}+G^{k l} \dot{G}_{l j}\right] \xi^{j}+\xi^{i} \xi^{j}\left\{\frac{1}{2} \frac{d}{d t}\left[G_{i k} H_{j}^{k}\right]+\frac{1}{4} \ddot{G}_{i j}\right\}+$ total derivative

This suggests that we define an analogue of the Christoffel symbol $\Gamma_{i j}^{k} \dot{q}^{i}$ of Riemannian geometry:

so that

$$
\gamma_{j}^{k}=\frac{1}{2}\left[-H_{j}^{k}+H^{k m} G_{j l} H_{m}^{l}+H^{k l} \dot{G}_{l j}\right]
$$

$$
-\dot{\xi}^{i} \xi^{j} G_{i k} H_{j}^{k}=\dot{\xi}^{i} G_{i k} \gamma_{j}^{k} \xi^{j}+\xi^{i} \xi^{j}\left\{\frac{1}{2} \frac{d}{d t}\left[G_{i k} H_{j}^{k}\right]+\frac{1}{4} \ddot{G}_{i j}\right\}+\text { total derivative }
$$

The point (which we prove below) is that although $\dot{\xi}^{i}$ does not transform as a vector , the "covariant time derivative"

$$
\stackrel{\circ}{\xi}=\dot{\xi}^{k}+\gamma_{j}^{k} \xi^{j}
$$

does. We do not attempt to define a covariant derivative along an arbitrary direction; only along the orbit of the Hamiltonian vector field.

We can now rewrite $\mathcal{S}_{1}$ in terms of this covariant derivative:

$$
\begin{aligned}
& \mathcal{S}_{1}=\int\left[\frac{1}{2} G_{i j} \dot{\xi}^{i} \dot{\xi}^{j}+\dot{\xi}^{i} G_{i k} \gamma_{j}^{k} \xi^{j}+\xi^{i} \xi^{j}\left\{\frac{1}{2} \frac{d}{d t}\left[G_{i k} H_{j}^{k}\right]+\frac{1}{4} \ddot{G}_{i j}\right\}+\frac{1}{2} \xi^{i} \xi^{j}\left\{-H_{i j}+H_{i}^{k} H_{j}^{l} G_{k l}\right\}\right] d t \\
& =\int\left[\frac{1}{2} G_{i j}\left\{\dot{\xi}^{i} \dot{\xi}^{j}+2 \dot{\xi}^{i} \gamma_{k}^{j} \xi^{k}\right\}+\xi^{i} \xi^{j}\left\{\frac{1}{2} \frac{d}{d t}\left[G_{i k} H_{j}^{k}\right]+\frac{1}{4} \ddot{G}_{i j}\right\}+\frac{1}{2} \xi^{i} \xi^{j}\left\{-H_{i j}+H_{i}^{k} H_{j}^{l} G_{k l}\right\}\right] d t \\
& =\int\left[\frac{1}{2} G_{i j}\left\{\dot{\xi}^{i} \dot{\xi}^{j}+\dot{\xi}^{i} \gamma_{k}^{j} \xi^{k}+\dot{\xi}^{j} \gamma_{k}^{i} \xi^{k}\right\}+\xi^{i} \xi^{j}\left\{\frac{1}{2} \frac{d}{d t}\left[G_{i k} H_{j}^{k}\right]+\frac{1}{4} \ddot{G}_{i j}\right\}+\frac{1}{2} \xi^{i} \xi^{j}\left\{-H_{i j}+H_{i}^{k} H_{j}^{l} G_{k l}\right\}\right] d t \\
& =\int\left[\frac{1}{2} G_{i j}\left\{\dot{\xi}^{i} \dot{\xi}^{j}+\dot{\xi}^{i} \gamma_{k}^{j} \xi^{k}+\gamma_{k}^{i} \xi^{k} \dot{\xi}^{j}\right\}+\xi^{i} \xi^{j}\left\{\frac{1}{2} \frac{d}{d t}\left[G_{i k} H_{j}^{k}\right]+\frac{1}{4} \ddot{G}_{i j}\right\}+\frac{1}{2} \xi^{i} \xi^{j}\left\{-H_{i j}+H_{i}^{k} H_{j}^{l} G_{k l}\right\}\right] d t \\
& =\int\left[\frac{1}{2} G_{i j} \dot{\circ} \dot{\xi}-\frac{1}{2} G_{k l} \gamma_{i}^{k} \gamma_{j}^{l} \xi^{i} \xi^{j}+\xi^{i} \xi^{j}\left\{\frac{1}{2} \frac{d}{d t}\left[G_{i k} H_{j}^{k}\right]+\frac{1}{4} \ddot{G}_{i j}\right\}+\frac{1}{2} \xi^{i} \xi^{j}\left\{-H_{i j}+H_{i}^{k} H_{j}^{l} G_{k l}\right\}\right] d t
\end{aligned}
$$

Thus 


$$
\mathcal{S}=\int\left[\frac{1}{2} G_{i j} \stackrel{\stackrel{i}{\circ}^{\circ}}{\xi}-\xi^{i} \xi^{j} \frac{1}{2}\left\{G_{k l} \gamma_{i}^{k} \gamma_{j}^{l}-\frac{d}{d t}\left[G_{i k} H_{j}^{k}\right]-\frac{1}{2} \ddot{G}_{i j}+H_{i j}-H_{i}^{k} H_{j}^{l} G_{k l}\right\}\right] d t
$$

The symmetric part of the quantity in the curly brackets is an analogue of curvature.

$$
\mathcal{S}_{1}=\int\left[\frac{1}{2} G_{i j} \stackrel{\circ}{\xi} \stackrel{\circ}{\xi}-\frac{1}{2} \xi^{i} \xi^{j} \mathcal{R}_{i j}\right] d t
$$

Rewriting time derivatives as Poisson Brackets, we can express it in terms of the first four derivatives of the hamiltonian:

$$
\mathcal{R}_{i j}=G_{k l} \gamma_{i}^{k} \gamma_{j}^{l}-\frac{1}{2}\left\{H, G_{i k} H_{j}^{k}+G_{j k} H_{i}^{k}\right\}-\frac{1}{2}\left\{H,\left\{H, G_{i j}\right\}\right\}+H_{i j}-H_{i}^{k} H_{j}^{l} G_{k l}
$$

The trace

$$
\mathcal{R}=H^{i j} \mathcal{R}_{i j}
$$

plays the role of the Ricci tensor. There is no notion of Ricci scalar in general Hamiltonian mechanics. But, it makes sense to integrate this w.r.t. the Boltzmann measure

$$
\mathfrak{R}(H)=\int \mathcal{R} d \mu_{H}
$$

to give a functional of the hamiltonian. We will see that this reduces to the integral of the Ricci scalar over a Riemannian manifold.

3.1. The Transformation of $\gamma_{j}^{i}$ and $\mathcal{R}_{i j}$. Recall that $\xi^{i}$ transforms as the components of a vector field:

but not its time derivative:

$$
\tilde{\xi}^{i}=\frac{\partial \tilde{q}^{i}}{\partial q^{j}} \xi^{j}
$$

$$
\begin{gathered}
\frac{d}{d t} \tilde{\xi}^{i}=\frac{d}{d t}\left[\frac{\partial \tilde{q}^{i}}{\partial q^{j}} \xi^{j}\right] \\
\dot{\tilde{\xi}}^{i}=\frac{\partial \tilde{q}^{i}}{\partial q^{j}} \dot{\xi}^{j}+\frac{\partial^{2} \tilde{q}^{i}}{\partial q^{k} \partial q^{j}} \dot{q}^{k} \xi^{j}
\end{gathered}
$$

The inhomogeneous term in the covariant time derivative

$$
\stackrel{\circ}{\xi}^{i}=\dot{\xi}^{i}+\gamma_{j}^{k} \xi^{j}
$$

is cancelled if the symbols $\gamma_{j}^{i}$ transform as

For,

$$
\tilde{\gamma}_{j}^{i}=\frac{\partial \tilde{q}^{i}}{\partial q^{k}} \gamma_{l}^{k} \frac{\partial q^{l}}{\partial \tilde{q}^{j}}-\frac{\partial q^{l}}{\partial \tilde{q}^{j}} \frac{\partial^{2} \tilde{q}^{i}}{\partial q^{k} \partial q^{l}} \dot{q}^{k}
$$

$$
\stackrel{\circ^{i}}{\tilde{\xi}}=\dot{\tilde{\xi}}^{i}+\tilde{\gamma}_{j}^{i} \tilde{\xi}^{j}
$$




$$
\begin{aligned}
=\frac{\partial \tilde{q}^{i}}{\partial q^{j}} \dot{\xi}^{j}+\frac{\partial^{2} \tilde{q}^{i}}{\partial q^{k} \partial q^{j}} \dot{q}^{k} \xi^{j} & +\left\{\frac{\partial \tilde{q}^{i}}{\partial q^{k}} \gamma_{l}^{k} \frac{\partial q^{l}}{\partial \tilde{q}^{j}}-\frac{\partial q^{l}}{\partial \tilde{q}^{j}} \frac{\partial^{2} \tilde{q}^{i}}{\partial q^{k} \partial q^{l}} \dot{q}^{k}\right\} \frac{\partial \tilde{q}^{j}}{\partial q^{m}} \xi^{m} \\
& =\frac{\partial \tilde{q}^{i}}{\partial q^{j}}\left[\dot{\xi}^{j}+\gamma_{l}^{j} \xi^{l}\right]
\end{aligned}
$$

Using Hamilton's equations, we can write the required transformation law as

$$
\tilde{\gamma}_{j}^{i}=\frac{\partial \tilde{q}^{i}}{\partial q^{k}} \gamma_{l}^{k} \frac{\partial q^{l}}{\partial \tilde{q}^{j}}-\frac{\partial q^{l}}{\partial \tilde{q}^{j}} \frac{\partial^{2} \tilde{q}^{i}}{\partial q^{k} \partial q^{l}} H^{k}
$$

For later reference we rewrite this by a relabelling of indices as

$$
\tilde{\gamma}_{k}^{l}=\frac{\partial q^{c}}{\partial \tilde{q}^{k}} \frac{\partial \tilde{q}^{l}}{\partial q^{a}} \gamma_{c}^{a}-\frac{\partial q^{c}}{\partial \tilde{q}^{k}} \frac{\partial^{2} \tilde{q}^{l}}{\partial q^{c} \partial q^{a}} H^{a}
$$

Proposition. $\gamma_{k}^{l}$ transforms as above. So $\stackrel{\circ}{\xi}$ and $\mathcal{R}_{i j}$ transform as tensors.

Proof We need the transformations of $H_{k}^{l}, P_{k}^{l} \equiv H^{l j} G_{k i} H_{j}^{i}, G_{k j} \dot{H}^{i k}$.

The Transformation of $H_{k}^{l}$. Recall that

$$
\tilde{H}_{j}=H_{b} \frac{\partial q^{b}}{\partial \tilde{q}^{j}}+H^{a} \tilde{p}_{k} \frac{\partial^{2} \tilde{q}^{k}}{\partial q^{c} \partial q^{a}} \frac{\partial q^{c}}{\partial \tilde{q}^{j}}
$$

By differentiating w.r.t. to $\tilde{p}_{i}$ we get the transformation of $\tilde{H}_{j}^{i}$ :

$$
\tilde{H}_{j}^{i}=\frac{\partial q^{b}}{\partial \tilde{q}^{j}} \frac{\partial \tilde{q}^{i}}{\partial q^{a}} H_{b}^{a}+\frac{\partial q^{c}}{\partial \tilde{q}^{j}} \frac{\partial^{2} \tilde{q}^{i}}{\partial q^{c} \partial q^{a}} H^{a}+\frac{\partial q^{c}}{\partial \tilde{q}^{j}} \frac{\partial \tilde{q}^{i}}{\partial q^{a}} \frac{\partial^{2} \tilde{q}^{k}}{\partial q^{c} \partial q^{b}} H^{a b} \tilde{p}_{k}
$$

Relabelling indices (for later use)

$$
\tilde{H}_{k}^{l}=\frac{\partial q^{c}}{\partial \tilde{q}^{k}} \frac{\partial \tilde{q}^{l}}{\partial q^{a}} H_{c}^{a}+\frac{\partial q^{c}}{\partial \tilde{q}^{k}} \frac{\partial^{2} \tilde{q}^{l}}{\partial q^{c} \partial q^{a}} H^{a}+\frac{\partial q^{b}}{\partial \tilde{q}^{k}} \frac{\partial \tilde{q}^{l}}{\partial q^{d}} \frac{\partial^{2} \tilde{q}^{m}}{\partial q^{b} \partial q^{c}} H^{d c} \tilde{p}_{m}
$$

The Transformation of $P_{k}^{l}$.

$$
\tilde{P}_{k}^{l}=\tilde{H}^{l j} \tilde{G}_{k i}\left\{\frac{\partial q^{b}}{\partial \tilde{q}^{j}} \frac{\partial \tilde{q}^{i}}{\partial q^{a}} H_{b}^{a}+\frac{\partial q^{c}}{\partial \tilde{q}^{j}} \frac{\partial^{2} \tilde{q}^{i}}{\partial q^{c} \partial q^{a}} H^{a}+\frac{\partial q^{c}}{\partial \tilde{q}^{j}} \frac{\partial \tilde{q}^{i}}{\partial q^{a}} \frac{\partial^{2} \tilde{q}^{m}}{\partial q^{c} \partial q^{b}} H^{a b} \tilde{p}_{m}\right\}
$$

Note that

so that

$$
\tilde{H}^{l j} \tilde{G}_{k i} \frac{\partial q^{c}}{\partial \tilde{q}^{j}} \frac{\partial \tilde{q}^{i}}{\partial q^{a}}=H^{d c} G_{n a} \frac{\partial \tilde{q}^{l}}{\partial q^{d}} \frac{\partial q^{n}}{\partial \tilde{q}^{k}}
$$

$$
\begin{gathered}
\tilde{P}_{k}^{l}=H^{m b} G_{n a} H_{b}^{a} \frac{\partial \tilde{q}^{l}}{\partial q^{m}} \frac{\partial q^{n}}{\partial \tilde{q}^{k}}+\frac{\partial \tilde{q}^{l}}{\partial q^{m}} H^{m c} \tilde{G}_{k i} \frac{\partial^{2} \tilde{q}^{i}}{\partial q^{c} \partial q^{a}} H^{a}+H^{d c} G_{n a} \frac{\partial \tilde{q}^{l}}{\partial q^{d}} \frac{\partial q^{n}}{\partial \tilde{q}^{k}} \frac{\partial^{2} \tilde{q}^{m}}{\partial q^{c} \partial q^{b}} H^{a b} \tilde{p}_{m} \\
=P_{n}^{m} \frac{\partial \tilde{q}^{l}}{\partial q^{m}} \frac{\partial q^{n}}{\partial \tilde{q}^{k}}+\frac{\partial \tilde{q}^{l}}{\partial q^{m}} H^{m c} \tilde{G}_{k i} \frac{\partial^{2} \tilde{q}^{i}}{\partial q^{c} \partial q^{a}} H^{a}+H^{d c} \frac{\partial \tilde{q}^{l}}{\partial q^{d}} \frac{\partial q^{b}}{\partial \tilde{q}^{k}} \frac{\partial^{2} \tilde{q}^{m}}{\partial q^{c} \partial q^{b}} \tilde{p}_{m}
\end{gathered}
$$


CURVATURE IN HAMILTONIAN MECHANICS AND THE EINSTEIN-MAXWELL-DILATON ACTIOIA

The Transformation of $G_{k i} \dot{H}^{i l}$.

$$
\begin{aligned}
& \tilde{G}_{k i} \dot{\tilde{H}}^{i l}=\tilde{G}_{k i} \frac{d}{d t}\left[\frac{\partial \tilde{q}^{i}}{\partial q^{a}} \frac{\partial \tilde{q}^{l}}{\partial q^{c}} H^{a c}\right] \\
& =\tilde{G}_{k i} \frac{\partial \tilde{q}^{i}}{\partial q^{a}} \frac{\partial \tilde{q}^{l}}{\partial q^{c}} \dot{H}^{a c}+\tilde{G}_{k i} \frac{\partial \tilde{q}^{i}}{\partial q^{a}} \frac{\partial^{2} \tilde{q}^{l}}{\partial q^{c} \partial q^{b}} \dot{q}^{b} H^{a c}+\tilde{G}_{k i} \frac{\partial^{2} \tilde{q}^{i}}{\partial q^{a} \partial q^{b}} \frac{\partial \tilde{q}^{l}}{\partial q^{c}} \dot{q}^{b} H^{a c} \\
& =\tilde{G}_{k i} \frac{\partial \tilde{q}^{i}}{\partial q^{a}} \frac{\partial \tilde{q}^{l}}{\partial q^{c}} \dot{H}^{a c}+\frac{\partial^{2} \tilde{q}^{l}}{\partial q^{c} \partial q^{b}} \dot{q}^{b}\left\{H^{a c} \tilde{G}_{k i} \frac{\partial \tilde{q}^{i}}{\partial q^{a}}\right\}+\tilde{G}_{k i} \frac{\partial^{2} \tilde{q}^{i}}{\partial q^{a} \partial q^{b}} \frac{\partial \tilde{q}^{l}}{\partial q^{c}} \dot{q}^{b} H^{a c} \\
& =\tilde{G}_{k i} \frac{\partial \tilde{q}^{i}}{\partial q^{a}} \frac{\partial \tilde{q}^{l}}{\partial q^{c}} H^{a c}+\frac{\partial^{2} \tilde{q}^{l}}{\partial q^{c} \partial q^{b}} \dot{q}^{b}\left\{H^{a c} G_{a d} \frac{\partial q^{d}}{\partial \tilde{q}^{k}}\right\}+\tilde{G}_{k i} \frac{\partial^{2} \tilde{q}^{i}}{\partial q^{a} \partial q^{b}} \frac{\partial \tilde{q}^{l}}{\partial q^{c}} \dot{q}^{b} H^{a c} \\
& =\tilde{G}_{k i} \frac{\partial \tilde{q}^{i}}{\partial q^{a}} \frac{\partial \tilde{q}^{l}}{\partial q^{c}} \dot{H}^{a c}+\frac{\partial^{2} \tilde{q}^{l}}{\partial q^{c} \partial q^{b}} \dot{q}^{b} \frac{\partial q^{c}}{\partial \tilde{q}^{k}}+\tilde{G}_{k i} \frac{\partial^{2} \tilde{q}^{i}}{\partial q^{a} \partial q^{b}} \frac{\partial \tilde{q}^{l}}{\partial q^{c}} \dot{q}^{b} H^{a c} \\
& =\tilde{G}_{k i} \frac{\partial \tilde{q}^{i}}{\partial q^{a}} \frac{\partial \tilde{q}^{l}}{\partial q^{c}} \dot{H}^{a c}+\frac{\partial^{2} \tilde{q}^{l}}{\partial q^{c} \partial q^{a}} \frac{\partial q^{c}}{\partial \tilde{q}^{k}} \dot{q}^{a}+\tilde{G}_{k i} \frac{\partial^{2} \tilde{q}^{i}}{\partial q^{a} \partial q^{b}} \frac{\partial \tilde{q}^{l}}{\partial q^{c}} \dot{q}^{b} H^{a c} \\
& =\tilde{G}_{k i} \frac{\partial \tilde{q}^{i}}{\partial q^{a}} \frac{\partial \tilde{q}^{l}}{\partial q^{c}} \dot{H}^{a c}+\frac{\partial^{2} \tilde{q}^{l}}{\partial q^{c} \partial q^{a}} \frac{\partial q^{c}}{\partial \tilde{q}^{k}} H^{a}+\tilde{G}_{k i} \frac{\partial^{2} \tilde{q}^{i}}{\partial q^{a} \partial q^{b}} \frac{\partial \tilde{q}^{l}}{\partial q^{c}} H^{a} H^{a c} \\
& =\frac{\partial q^{b}}{\partial \tilde{q}^{k}} \frac{\partial \tilde{q}^{l}}{\partial q^{c}} G_{b a} \dot{H}^{a c}+\frac{\partial^{2} \tilde{q}^{l}}{\partial q^{c} \partial q^{a}} \frac{\partial q^{c}}{\partial \tilde{q}^{k}} H^{a}+\tilde{G}_{k i} \frac{\partial^{2} \tilde{q}^{i}}{\partial q^{a} \partial q^{b}} \frac{\partial \tilde{q}^{l}}{\partial q^{c}} H^{a} H^{a c}
\end{aligned}
$$

where, we use Hamilton's equation $\dot{q}^{a}=H^{a}$.

Relabeling $c \rightarrow a, a \rightarrow b, b \rightarrow c$ in the first term and $c \rightarrow m, a \rightarrow c, b \rightarrow a$, in the last term (for later use),

$$
\tilde{G}_{k i} \dot{\tilde{H}}^{i l}=\frac{\partial q^{c}}{\partial \tilde{q}^{k}} \frac{\partial \tilde{q}^{l}}{\partial q^{a}} G_{c b} \dot{H}^{b a}+\frac{\partial^{2} \tilde{q}^{l}}{\partial q^{c} \partial q^{a}} \frac{\partial q^{c}}{\partial \tilde{q}^{k}} H^{a}+\tilde{G}_{k i} \frac{\partial^{2} \tilde{q}^{i}}{\partial q^{c} \partial q^{a}} \frac{\partial \tilde{q}^{l}}{\partial q^{m}} H^{a} H^{c m}
$$

So consider the linear combination

$$
\begin{gathered}
A \tilde{H}_{k}^{l}+B \tilde{P}_{k}^{l}+C \tilde{G}_{k i} \dot{\tilde{G}}^{i l} \\
=A\left\{\frac{\partial q^{c}}{\partial \tilde{q}^{k}} \frac{\partial \tilde{q}^{l}}{\partial q^{a}} H_{c}^{a}+\frac{\partial q^{c}}{\partial \tilde{q}^{k}} \frac{\partial^{2} \tilde{q}^{l}}{\partial q^{c} \partial q^{a}} H^{a}+\frac{\partial q^{b}}{\partial \tilde{q}^{k}} \frac{\partial \tilde{q}^{l}}{\partial q^{d}} \frac{\partial^{2} \tilde{q}^{m}}{\partial q^{b} \partial q^{c}} H^{d c} \tilde{p}_{m}\right\} \\
+B\left\{P_{c}^{a} \frac{\partial \tilde{q}^{l}}{\partial q^{a}} \frac{\partial q^{c}}{\partial \tilde{q}^{k}}+\frac{\partial \tilde{q}^{l}}{\partial q^{m}} H^{m c} \tilde{G}_{k i} \frac{\partial^{2} \tilde{q}^{i}}{\partial q^{c} \partial q^{a}} H^{a}+H^{d c} \frac{\partial \tilde{q}^{l}}{\partial q^{d}} \frac{\partial q^{b}}{\partial \tilde{q}^{k}} \frac{\partial^{2} \tilde{q}^{m}}{\partial q^{c} \partial q^{b}} \tilde{p}_{m}\right\} \\
+C\left\{\frac{\partial q^{c}}{\partial \tilde{q}^{k}} \frac{\partial \tilde{q}^{l}}{\partial q^{a}} G_{c b} \dot{H}^{b a}+\frac{\partial^{2} \tilde{q}^{l}}{\partial q^{c} \partial q^{a}} \frac{\partial q^{c}}{\partial \tilde{q}^{k}} H^{a}+\tilde{G}_{k i} \frac{\partial^{2} \tilde{q}^{i}}{\partial q^{c} \partial q^{a}} \frac{\partial \tilde{q}^{l}}{\partial q^{m}} H^{a} H^{c m}\right\}
\end{gathered}
$$

If we choose

$$
A+C=-1, \quad B+C=0, \quad A+B=0 \Longrightarrow A=-\frac{1}{2}=C, \quad B=\frac{1}{2}
$$

we get the transformation law

$\frac{1}{2}\left[-\tilde{H}_{k}^{l}+\tilde{P}_{k}^{l}-\tilde{G}_{k i} \dot{\tilde{G}}^{i l}\right]=\frac{\partial q^{c}}{\partial \tilde{q}^{k}} \frac{\partial \tilde{q}^{l}}{\partial q^{a}} \frac{1}{2}\left[-H_{c}^{a}+P_{c}^{a}-G_{c b} \dot{H}^{b a}\right]-\frac{\partial q^{c}}{\partial \tilde{q}^{k}} \frac{\partial^{2} \tilde{q}^{l}}{\partial q^{c} \partial q^{a}} H^{a}$ 
So we can choose

$$
\gamma_{c}^{a}=\frac{1}{2}\left[-H_{c}^{a}+P_{c}^{a}-G_{c b} \dot{H}^{b a}\right]=\frac{1}{2}\left[-H_{c}^{a}+P_{c}^{a}+\dot{G}_{c b} H^{b a}\right]
$$

(where we used $\dot{G}_{c b} H^{b a}+G_{c b} \dot{H}^{b a}=0$ ) to get the transformation law

$$
\tilde{\gamma}_{k}^{l}=\frac{\partial q^{c}}{\partial \tilde{q}^{k}} \frac{\partial \tilde{q}^{l}}{\partial q^{a}} \gamma_{c}^{a}-\frac{\partial q^{c}}{\partial \tilde{q}^{k}} \frac{\partial^{2} \tilde{q}^{l}}{\partial q^{c} \partial q^{a}} H^{a}
$$

This is what we wanted.

\section{Comparison With Riemannian Geometry}

We must show that the above formula (3.1) reduces to the usual one for curvature in Riemannian geometry. In this section, unlike before, we raise and lower indices using the metric tensor.

If $H=\frac{1}{2} g^{i j} p_{i} p_{j}$ Hamilton's equations reduce to the geodesic equation[13] . Also,

$$
\begin{gathered}
H^{i j}=g^{i j} \\
H_{i}^{j}=\partial_{i} g^{j k} p_{k}
\end{gathered}
$$

Recalling the formula for the Christoffel symbols,

$$
\Gamma_{j k}^{i}=\frac{1}{2} g^{i m}\left[\partial_{k} g_{j m}+\partial_{j} g_{k m}-\partial_{m} g_{j k}\right]
$$

we can rewrite them in terms of the contra-variant metric tensor

$$
\begin{gathered}
\Gamma_{j k}^{i} \dot{q}^{k}=\frac{1}{2} g^{i m}\left[\partial_{k} g_{j m}+\partial_{j} g_{k m}-\partial_{m} g_{j k}\right] \dot{q}^{k} \\
=\frac{1}{2} g^{i m}\left[\dot{g}_{j m}+\left\{-g_{k a} g_{m b} \partial_{j} g^{a b}+g_{j a} g_{k b} \partial_{m} g^{a b}\right\} \dot{q}^{k}\right] \\
=\frac{1}{2}\left[g^{i k} \dot{g}_{j k}+\left\{-p_{a} \partial_{j} g^{a i}+g_{j a} p_{b} g^{i m} \partial_{m} g^{a b}\right\}\right]
\end{gathered}
$$

This is a particular case of the general formula

$$
\gamma_{j}^{i}=\frac{1}{2}\left[H^{i k} \dot{G}_{k j}-H_{j}^{i}+G_{j l} H_{m}^{l} H^{i m}\right]
$$

so that $\gamma_{j}^{i}$ reduces to $\Gamma_{j k}^{i} \dot{q}^{k}$ in Riemannian geometry.

The Riemann tensor is

$$
\begin{gathered}
R_{i j k}^{l}=\partial_{j} \Gamma_{i k}^{l}-\partial_{k} \Gamma_{i j}^{l}+\Gamma_{j m}^{l} \Gamma_{i k}^{m}-\Gamma_{k m}^{l} \Gamma_{i j}^{m} \\
R_{l i j k}=g_{l n} R_{i j k}^{n}
\end{gathered}
$$

To compare the curvatures, it is convenient to choose Riemann normal coordinates in which the first derivative of the metric is zero at the chosen point. $(\approx$ denotes equality in Riemannian normal co-ordinates up to higher order terms.)

$$
\begin{gathered}
g_{i j} \approx \delta_{i j} \\
R_{i k l m} \approx \frac{1}{2}\left(\partial_{k} \partial_{l} g_{i m}+\partial_{i} \partial_{m} g_{k l}-\partial_{k} \partial_{m} g_{i l}-\partial_{i} \partial_{l} g_{k m}\right)
\end{gathered}
$$




$$
R_{i k l j} \approx \frac{1}{2}\left(\partial_{k} \partial_{l} g_{i j}+\partial_{i} \partial_{j} g_{k l}-\partial_{k} \partial_{j} g_{i l}-\partial_{i} \partial_{l} g_{k j}\right)
$$

On the other hand,

$$
\mathcal{R}_{i j} \approx-\frac{1}{2}\left(\dot{H}_{j}^{i}+\dot{H}_{i}^{j}\right)-\frac{1}{2} \ddot{G}_{i j}+H_{i j}
$$

In Riemannian geometry,

$$
\begin{gathered}
\dot{G}_{i j} \equiv\left\{H, G_{i j}\right\}=H^{k} \partial_{k} g_{i j} \\
\ddot{G}_{i j}=H^{m} \partial_{m}\left[H^{n} \partial_{n} g_{i j}\right]-H_{m} \frac{\partial}{\partial p_{m}}\left[H^{n} \partial_{n} g_{i j}\right]=H^{m} H^{n} \partial_{m} \partial_{n} g_{i j}+H^{m} H_{m}^{n} \partial_{n} g_{i j}-H_{m} H^{m n} \partial_{n} g_{i j} \\
\dot{H}_{j}^{i} \equiv\left\{H, \partial_{i} g^{j k} p_{k}\right\}=H^{m} \partial_{m} \partial_{i} g^{j k} p_{k}-H_{k} \partial_{i} g^{j k}
\end{gathered}
$$

so that in normal co-ordinates

$$
\begin{gathered}
\ddot{G}_{i j} \approx p_{k} p_{l} \partial_{k} \partial_{l} g_{i j} \\
\dot{H}_{j}^{i} \approx p_{k} p_{m} \partial_{m} \partial_{i} g^{j k} \approx-p_{k} p_{l} \partial_{l} \partial_{i} g_{j k} \\
H_{i j}=\frac{1}{2} \partial_{i} \partial_{j} g^{k l} p_{k} p_{l} \approx-\frac{1}{2} p_{k} p_{l} \partial_{i} \partial_{j} g_{k l} \\
\mathcal{R}_{i j} \approx \frac{1}{2} p_{k} p_{l}\left(\partial_{l} \partial_{i} g_{j k}+\partial_{l} \partial_{j} g_{i k}\right)-\frac{1}{2} p_{k} p_{l} \partial_{k} \partial_{l} g_{i j}-\frac{1}{2} p_{k} p_{l} \partial_{i} \partial_{j} g_{k l}
\end{gathered}
$$

Thus

$$
\mathcal{R}_{i j} \approx-p_{k} p_{l} R_{i k l j}
$$

Since $\mathcal{R}_{i j}$ and $R_{i j k}^{l}$ are tensors we get the equality in general co-ordinates

Moreover

$$
\mathcal{R}_{i j}=-g_{i m} H^{k} H^{l} R_{k l j}^{m}, \quad H^{m}=g^{m n} p_{n}
$$

so that

$$
H^{i j} \mathcal{R}_{i j}=-H^{k} H^{l} R_{k l m}^{m}=H^{k} H^{l} R_{k m l}^{m}
$$

Note the Gaussian integrals

$$
\mathcal{R} \equiv H^{i j} \mathcal{R}_{i j}=H^{k} H^{l} R_{k l} .
$$

$$
\int e^{-\frac{1}{2} g^{i j} p_{i} p_{j}} \frac{d^{n} p}{[2 \pi]^{\frac{n}{2}}}=\sqrt{g}, \quad \int e^{-\frac{1}{2} g^{i j} p_{i} p_{j}} p_{k} p_{l} \frac{d^{n} p}{[2 \pi]^{\frac{n}{2}}}=\sqrt{g} g_{k l}
$$

Thus we get the Einstein-Hilbert Lagrangian for GR as the Boltzmann average of the Ricci tensor:

$$
\mathscr{R} \equiv \int \mathcal{R} e^{-H} \frac{d^{n} p}{[2 \pi]^{\frac{n}{2}}}=R \sqrt{g}, \quad R=R_{k l} g^{k l} .
$$

Thus there might be some merit in considering $\mathscr{R}(H)$ as a variational principle that determines the hamiltonian itself in the general case. 


\section{Adding a Magnetic Field and a Scalar Potential}

The typical hamiltonian of a point particle in physics is a polynomial of order two in the momenta; it describes its interaction with a gravitational electromagnetic and scalar field

We can compute,

$$
H=\frac{1}{2} g^{k l}\left[p_{k}-A_{k}\right]\left[p_{l}-A_{l}\right]+\phi
$$

$$
\begin{gathered}
H^{k}=g^{k l}\left[p_{l}-A_{l}\right] \\
H_{j}^{k}=\partial_{j} g^{k l}\left[p_{l}-A_{l}\right]-g^{k l} \partial_{j} A_{l} \\
H_{m}^{l}=\partial_{m} g^{l n}\left[p_{n}-A_{n}\right]-g^{l n} \partial_{m} A_{n} \\
H^{k l}=g^{k l}, \quad G_{k l}=g_{k l} \\
H^{k m} G_{j l} H_{m}^{l}=g^{k m} g_{j l}\left\{\partial_{m} g^{l n}\left[p_{n}-A_{n}\right]-g^{l n} \partial_{m} A_{n}\right\} \\
=-g^{k m}\left[\partial_{m} g_{j l}\right] g^{l n}\left[p_{n}-A_{n}\right]-g^{k m} \partial_{m} A_{j} \\
H^{k l} \dot{G}_{i j}=g^{k l} H^{n} \partial_{n} g_{k l}
\end{gathered}
$$

To proceed further we pass to the Riemann normal co-ordinates normal co-ordinates; also choose $A_{i}=0$ at the origin by a choice of gauge (but of course not the derivative $\left.\partial_{i} A_{j}\right)$.

and

$$
\gamma_{j}^{k} \approx \frac{1}{2}\left[\partial_{j} A_{k}-\partial_{k} A_{j}\right]=\frac{1}{2} F_{j k}
$$

$$
\begin{gathered}
\mathcal{R}_{i j} \approx \gamma_{i}^{k} \gamma_{j}^{k}-\frac{1}{2}\left\{\dot{H}_{j}^{i}+\dot{H}_{i}^{j}\right\}-\frac{1}{2} \ddot{G}_{i j}+H_{i j}-H_{i}^{k} H_{j}^{l} G_{k l} \\
\dot{H}_{j}^{i} \approx-p_{k} p_{l} \partial_{l} \partial_{i} g_{j k}-p_{k} \partial_{k} \partial_{j} A_{i} \\
\ddot{G}_{i j} \approx p_{k} p_{l} \partial_{k} \partial_{l} g_{i j} \\
H_{i j} \approx-\frac{1}{2} p_{k} p_{l} \partial_{i} \partial_{j} g_{k l}+g^{k l} \partial_{i} A_{k} \partial_{j} A_{l}-p_{k} \partial_{i} \partial_{j} A_{k}+\partial_{i} \partial_{j} \phi \\
H_{i}^{k} H_{j}^{l} G_{k l} \approx \partial_{i} A_{k} \partial_{j} A_{k} \\
-\frac{1}{2}\left\{\dot{H}_{j}^{i}+\dot{H}_{i}^{j}\right\}+H_{i j}-H_{i}^{k} H_{j}^{l} G_{k l} \approx \frac{1}{2} p_{k} p_{l}\left[\partial_{l} \partial_{i} g_{j k}+\partial_{l} \partial_{j} g_{i k}-\partial_{i} \partial_{j} g_{k l}\right]+ \\
p_{k}\left\{\frac{1}{2} \partial_{k} \partial_{j} A_{i}+\frac{1}{2} \partial_{k} \partial_{i} A_{j}-\partial_{i} \partial_{j} A_{k}\right\}+g^{k l} \partial_{i} A_{k} \partial_{j} A_{l}-\partial_{i} A_{k} \partial_{j} A_{k}+\partial_{i} \partial_{j} \phi
\end{gathered}
$$




$$
\begin{gathered}
=\frac{1}{2} p_{k} p_{l}\left[\partial_{l} \partial_{i} g_{j k}+\partial_{l} \partial_{j} g_{i k}-\partial_{i} \partial_{j} g_{k l}\right]+\frac{1}{2} p_{k}\left\{\partial_{j} F_{k i}+\partial_{i} F_{k j}\right\}+\partial_{i} \partial_{j} \phi \\
\mathcal{R}_{i j} \approx \frac{1}{4} F_{i k} F_{j k}+\frac{1}{2} p_{k} p_{l}\left[\partial_{l} \partial_{i} g_{j k}+\partial_{l} \partial_{j} g_{i k}-\partial_{i} \partial_{j} g_{k l}\right]+\frac{1}{2} p_{k}\left\{\partial_{j} F_{k i}+\partial_{i} F_{k j}\right\}+\partial_{i} \partial_{j} \phi
\end{gathered}
$$

In a general co-ordinate system, this is the tensorial equality

$$
\mathcal{R}_{i j}=-g_{i m} H^{k} H^{l} R_{k l j}^{m}+\frac{1}{4} F_{i k} F_{j l} g^{k l}+\frac{1}{2} H^{k}\left\{\partial_{j} F_{k i}+\partial_{i} F_{k j}\right\}+\partial_{i} \partial_{j} \phi
$$

Taking a trace

$$
\mathcal{R}=H^{k} H^{l} R_{k l}+\frac{1}{4} F_{i k} F_{j l} g^{k l} g^{i j}+H^{k} g^{i j} \partial_{i} F_{k j}+\Delta \phi
$$

5.1. An Action Principle for Fields. The integral over momentum with the Boltzmann weight now has an extra factor of $e^{-\phi}$ :

$$
\begin{gathered}
\int e^{-\left\{\frac{1}{2} g^{i j}\left[p_{i}-A_{i}\right]\left[p_{j}-A_{j}\right]+\phi\right\}} \frac{d^{n} p}{[2 \pi]^{\frac{n}{2}}}=e^{-\phi} \sqrt{g} \\
\int e^{-\left\{\frac{1}{2} g^{i j}\left[p_{i}-A_{i}\right]\left[p_{j}-A_{j}\right]+\phi\right\}}\left[p_{k}-A_{k}\right]\left[p_{l}-A_{l}\right] \frac{d^{n} p}{[2 \pi]^{\frac{n}{2}}}=e^{-\phi} \sqrt{g} g_{k l}
\end{gathered}
$$

Thus

$$
\mathscr{R} \equiv \int \mathcal{R} e^{-H} \frac{d^{n} p}{[2 \pi]^{\frac{n}{2}}}=\left[R+\frac{1}{4} F_{i k} F_{j l} g^{k l} g^{i j}+\Delta \phi\right] e^{-\phi} \sqrt{g}
$$

Similar actions also arise in string theory and in Kaluza-Klein theories; the scalar $\phi$ is the dilaton in that context.

If we make the field redefinition

and choose

$$
\tilde{g}_{i j}=e^{2 \alpha \phi} g_{i j}
$$

$$
\alpha=-\frac{1}{n-2}
$$

the action of the fields can be brought to the more conventional form (dropping a total derivative)

$$
\mathscr{R}=\sqrt{\tilde{g}} \tilde{R}+\frac{1}{4} F_{i k} F_{j l} \tilde{g}^{k l} \tilde{g}^{i j} \sqrt{\tilde{g}} e^{-\frac{2}{n-2} \phi}+\frac{2 n-1}{n-2} \sqrt{\tilde{g}} \tilde{g}^{i j} \partial_{i} \phi \partial_{j} \phi
$$

The parametrization of the original Hamiltonian is, for reference,

$$
H=\frac{1}{2} e^{-\frac{2}{n-2} \phi} \tilde{g}^{i j}\left[p_{i}-A_{i}\right]\left[p_{j}-A_{j}\right]+\phi .
$$

This action describes a scalar and a photon minimally coupled to the gravitational field, with an additional non-minimal coupling of the scalar to the photon. 


\section{Particular Cases}

6.1. The Free Particle. The simplest, but rather trivial, case is the hamiltonian of a particle moving on the real line

$$
H=\frac{1}{2} p^{2}
$$

Of course, the curvature is zero. The trajectories are straight lines. It is straightforward to get

$$
s_{T}\left(Q, Q^{\prime}\right)=\frac{\left(Q-Q^{\prime}\right)^{2}}{2 T}, \quad \sigma_{E}\left(Q, Q^{\prime}\right)=\sqrt{2 E}\left|Q-Q^{\prime}\right|
$$

They satisfy the HJ equations

$$
\frac{1}{2}\left[\partial_{Q} s_{T}\right]^{2}+\frac{\partial s_{T}}{\partial T}=0, \quad \frac{1}{2}\left[\partial_{Q} \sigma_{E}\right]^{2}=E
$$

6.2. The Harmonic Oscillator. The simplest non-Euclidean geometry is the sphere; it has constant positive curvature. The mechanical analogue is the simple harmonic oscillator

$$
H=\frac{1}{2}\left[p^{2}+\omega^{2} q^{2}\right]
$$

The Lagrangian sub-manifold (configuration space) $M$ is one-dimensional, just the real line $\mathbb{R}$. Even the real line is curved in our sense! It is constant and positive:

$$
\mathcal{R}_{11}=\omega^{2}
$$

In this is case, this is also the Ricci form. The phase space has finite volume in the Boltzmann measure; the induced volume element on the real line is the Gaussian.

$$
d q \int e^{-H} \frac{d p}{\sqrt{2 \pi}}=e^{-\frac{1}{2} \omega^{2} q^{2}} d q
$$

Given $E>0$, the set of allowed positions

$$
M_{E}=\{q \mid H(q, p)=E \text { for some } p\}=\left[-\frac{\sqrt{2 E}}{\omega}, \frac{\sqrt{2 E}}{\omega}\right]
$$

is just the interval $|q|<\frac{\sqrt{E}}{\omega}$; i.e., the major axis of the energy ellipse. Given two points $Q, Q^{\prime} \in M_{E}$ we have the solution to the eikonal equation

$$
\sigma_{E}\left(Q, Q^{\prime}\right)=\int_{Q^{\prime}}^{Q} \sqrt{2 E-\omega^{2} q^{2}} d q
$$

Geometrically, this is area of the region bounded by the energy ellipse, vertical axes at $Q, Q^{\prime}$ and the horizontal axis. $\sigma_{E}\left(Q, Q^{\prime}\right)$ is a metric (in the sense of topology) on the above interval. The maximum of $\sigma_{E}\left(Q, Q^{\prime}\right)$ occurs when $Q=-\frac{\sqrt{E}}{\omega}, Q^{\prime}=-\frac{\sqrt{E}}{\omega}$ and is equal to half the area of the energy ellipse. Thus,

$$
\sigma_{E}\left(Q, Q^{\prime}\right) \leq \pi \frac{E}{\omega}
$$


This is reminiscent of Myer's inequality in Riemannian geometry[1]. If the Ricci tensor is bounded below

$$
R_{i j} \xi^{i} \xi^{j} \geq \omega^{2} \xi^{i} \xi^{j} g_{i j}, \quad \omega>0
$$

the distance between any two points in the manifold is bounded as well:

$$
d\left(Q, Q^{\prime}\right) \leq \frac{\pi}{\omega} .
$$

Could there be a generalization of Myer's theorem to more general mechanical systems with a convex, time-symmetric Hamiltonian?

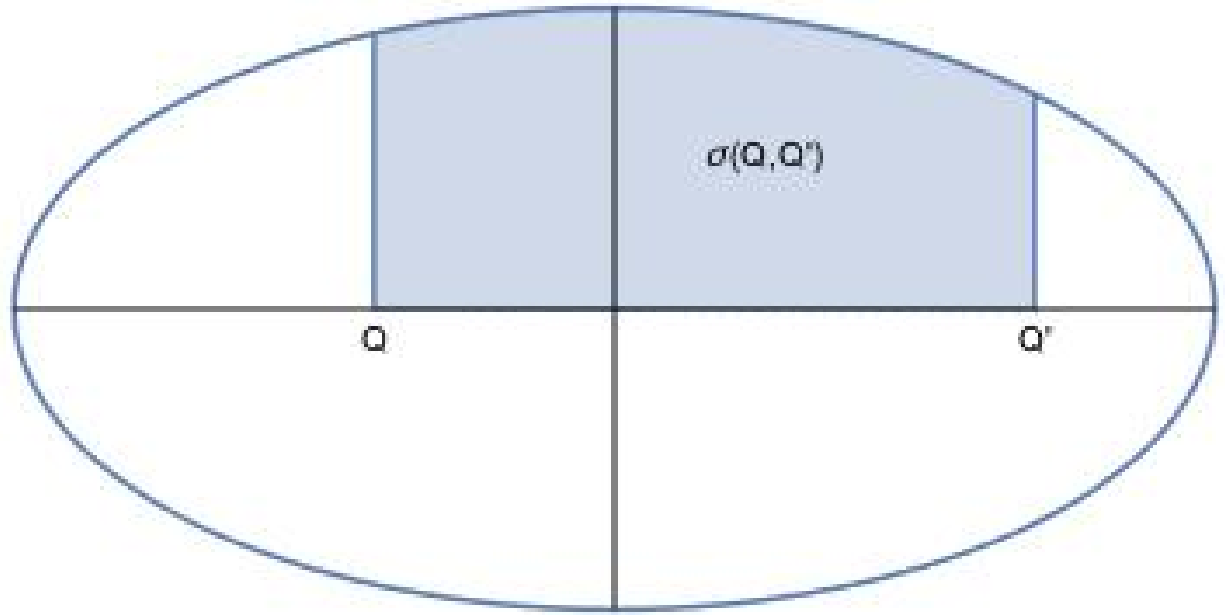

The inverted harmonic oscillator

$$
H=\frac{1}{2}\left[p^{2}-\omega^{2} q^{2}\right]
$$

has an unstable equilibrium point at $q=0=p$; it has constant negative curvature

$$
\mathcal{R}_{11}=-\omega^{2}
$$

and is the mechanical analogue of Lobachewski space.

6.3. Constant Magnetic Field. In the case of a particle moving on the plane we denote the co-ordinates by $z \equiv(x, y)$ instead of $q^{1}, q^{2}$. The Hamiltonian

$$
H=\frac{1}{2}\left[p_{x}+B y\right]^{2}+\frac{1}{2}\left[p_{y}-B x\right]^{2}
$$

corresponds to a particle in a constant magnetic field $B$ normal to the plane. (We choose units where the mass and charge are equal to one. So $B$ is just the cyclotron frequency.)

The curvature is constant and positive:

$$
\mathcal{R}_{i j}=\frac{1}{4} B^{2} \delta_{i j} .
$$

Hamilton's equations are equivalent to the Lorentz equations

$$
\ddot{x}=B \dot{y}, \quad \ddot{y}=-B \dot{x}
$$


It is instructive to find the action $s_{T}\left(Z, Z^{\prime}\right)$ of the solution satisfying the boundary conditions

$$
\begin{array}{cc}
x(0)=X^{\prime}, & x(T)=X \\
y(0)=Y^{\prime} & y(T)=Y
\end{array}
$$

This is a standard exercise in physics textbooks 14. After a long but straightforward calculation we get

$$
s_{T}\left(Z, Z^{\prime}\right)=\left[\frac{1}{4} B \cot \frac{B T}{2}\right]\left|Z-Z^{\prime}\right|^{2}-\frac{1}{2} B Z \times Z^{\prime}
$$

where the cross-product is $Z \times Z^{\prime}=X Y^{\prime}-Y X^{\prime}$.

We can verify directly that this satisfies the Hamilton-Jacobi equation

Its Legendre transform

$$
\frac{1}{2}\left[\partial_{X} s_{T}+B Y\right]^{2}+\frac{1}{2}\left[\partial_{Y} s_{T}-B X\right]^{2}+\frac{\partial s_{T}}{\partial T}=0
$$

$$
\begin{gathered}
\sigma_{E}\left(Z, Z^{\prime}\right)=\min _{T}\left[E T+s_{T}\left(Z, Z^{\prime}\right)\right] \\
=\frac{2 E}{B} \arcsin \left[\frac{B\left|Z^{\prime}-Z\right|}{2 \sqrt{2 E}}\right]+\frac{1}{2}\left|Z^{\prime}-Z\right| \sqrt{2 E-\frac{1}{4}\left|Z^{\prime}-Z\right|^{2} B^{2}}-\frac{1}{2} B Z \times Z
\end{gathered}
$$

Since the trajectory is a circle of radius $\frac{\sqrt{2 E}}{B}$, only points with $\left|Z-Z^{\prime}\right|<2 \frac{\sqrt{2 E}}{B}$ are connected by a smooth trajectory. Farther points would be connected by stitching together piecewise-circular segments. The above formula describes only one such segment.

Again we can verify directly that the stationary HJ equation is satisfied:

$$
\frac{1}{2}\left[\partial_{X} \sigma_{E}+B Y\right]^{2}+\frac{1}{2}\left[\partial_{Y} \sigma_{E}-B X\right]^{2}=E
$$

In units where $E=\frac{1}{2}$, (i.e., unit velocity)

$$
\sigma\left(Z, Z^{\prime}\right)=\frac{1}{B} \arcsin \left[\frac{B\left|Z^{\prime}-Z\right|}{2}\right]+\frac{1}{2}\left|Z^{\prime}-Z\right| \sqrt{1-\frac{1}{4}\left|Z^{\prime}-Z\right|^{2} B^{2}}-\frac{1}{2} B Z \times Z^{\prime}
$$

We can understand each of these terms geometrically. The trajectory is a circle of radius $\frac{\sqrt{2 E}}{B}$ connecting $Z$ to $Z^{\prime}$. If $B>0$ it is described in a counter-clockwise direction. Let $C$ be the center and let $M$ be the point halfway on the chord $Z Z^{\prime}$. Consider the right triangle $C M Z^{\prime}$. The lengths of its sides are

$$
\left|C Z^{\prime}\right|=\frac{1}{B}, \quad\left|M Z^{\prime}\right|=\frac{1}{2}\left|Z^{\prime}-Z\right|, \quad|C M|=\frac{1}{B} \sqrt{1-\frac{1}{4}\left|Z^{\prime}-Z\right|^{2} B^{2}}
$$

The half-angle at the center is

$$
\widehat{M C Z^{\prime}}=\arcsin \left[\frac{B\left|Z^{\prime}-Z\right|}{2}\right]=\widehat{M C Z}
$$

Thus each term in $\sigma\left(Z, Z^{\prime}\right)$ has a meaning of an area (times $B$ ), as illustrated in the figure: 
- the first term is the area of the circular arc of angle $\widehat{M C Z}$ (Blue)

- the second term is the area of the right triangle $C M Z^{\prime}$ (Yellow)

- the third term subtracts the area of the triangle $O Z Z^{\prime}$ (Red)

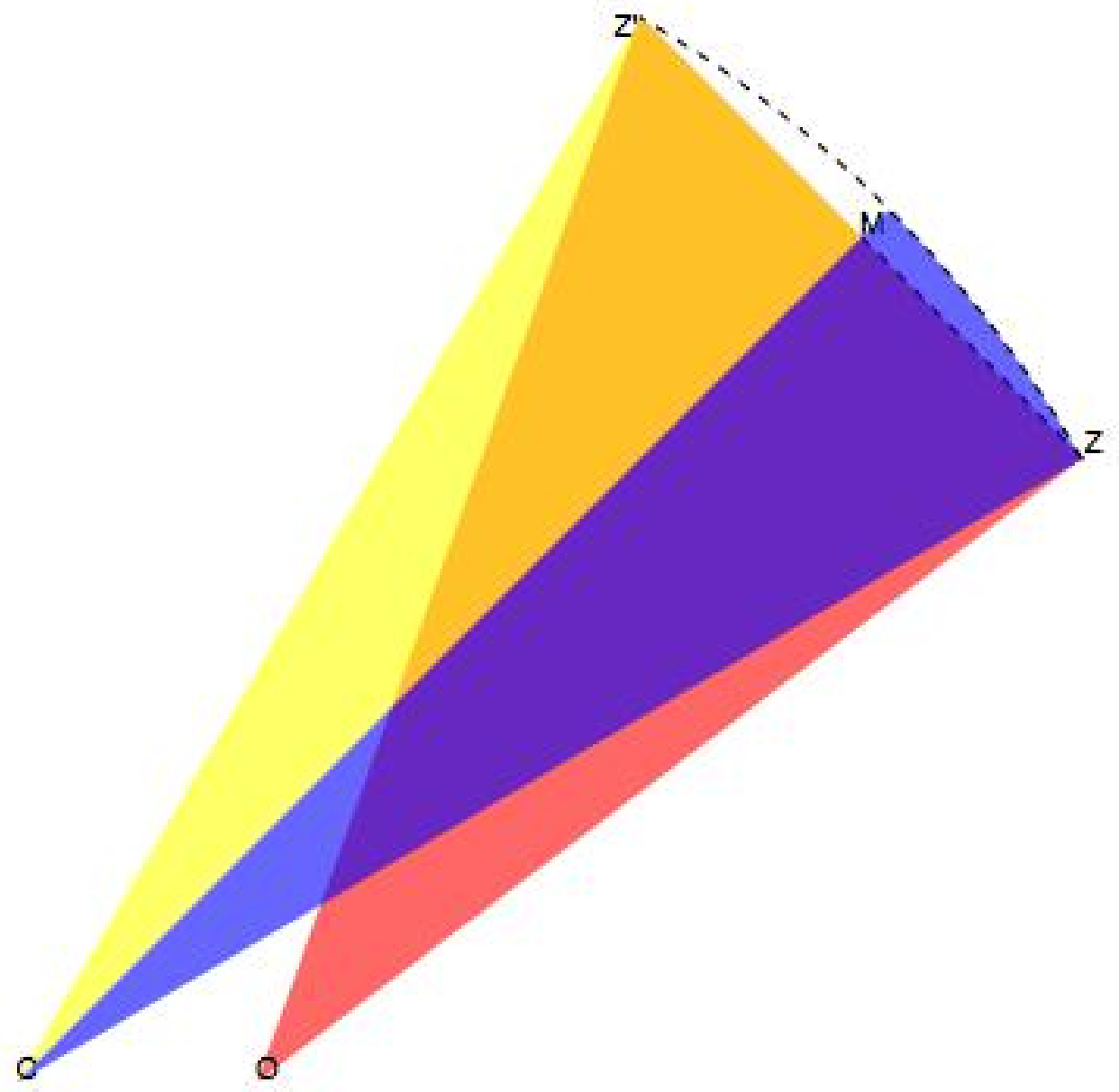

6.4. Magnetic Field Plus Quadratic potential. We can combine the above two cases and add an extra dimension to get

$$
H=\frac{1}{2}\left[p_{x}+B y\right]^{2}+\frac{1}{2}\left[p_{y}-B x\right]^{2}+\frac{1}{2} p_{z}^{2}+\phi
$$

where $\phi$ is a positive quadratic form in $x, y, z$. This describes a particle in a Penning trap or the immediate vicinity of a Lagrange point in the circular restricted three-body problem. (In the co-rotating frame of the primary bodies, there is a Coriolis force which is mathematically identical to the force due to a constant magnetic field normal to the plane of rotation.) The curvature

$$
\mathcal{R}_{i j}=\frac{1}{4} F_{i k} F_{j l} g^{k l}+\partial_{i} \partial_{j} \phi
$$

can be written conveniently in the co-ordinate system which diagonalizes $\partial_{i} \partial_{j} \phi$ 


$$
\begin{gathered}
\partial_{i} \partial_{j} \phi=\left(\begin{array}{ccc}
k_{1} & 0 & 0 \\
0 & k_{2} & 0 \\
0 & 0 & k_{3}
\end{array}\right) \\
F_{i j}=\left(\begin{array}{ccc}
0 & B & 0 \\
-B & 0 & 0 \\
0 & 0 & 0
\end{array}\right) \\
\mathcal{R}_{i j}=\left(\begin{array}{ccc}
k_{1}+\frac{1}{4} B^{2} & 0 & 0 \\
0 & k_{2}+\frac{1}{4} B^{2} & 0 \\
0 & 0 & k_{3}
\end{array}\right)
\end{gathered}
$$

If $\phi$ is harmonic (e.g., an electrostatic field as in the Penning trap or a Newtonian Gravitational field as in the three-body problem)

$$
k_{1}+k_{2}+k_{3}=0 .
$$

It is well known that such a harmonic potential $\phi$ does not have a stable equilibrium as at least one of the $k_{i}$ must be negative. Adding a strong enough magnetic field can stabilize such a potential; this is the idea behind the Penning trap and the surprising stability of the Lagrange points $L_{4}$ and $L_{5}$.

Whether harmonic or not, the case

$$
k_{3}>0, \quad k_{1}<0, \quad k_{2}<0, \quad \frac{1}{2} B^{2}>\sqrt{k_{1} k_{2}}+\frac{\left|k_{1}\right|+\left|k_{2}\right|}{2}
$$

is known to be stable[15].

In this case, if the curvature is positive,

$$
k_{3}>0, \quad \frac{1}{4} B^{2}>\left|k_{1}\right|, \quad \frac{1}{4} B^{2}>\left|k_{2}\right|
$$

it follows that $\frac{1}{4} B^{2}$ is also greater than the average of the geometric and arithmetic means of the r.h.s.:

$$
\frac{1}{4} B^{2}>\frac{1}{2}\left[\sqrt{k_{1} k_{2}}+\frac{\left|k_{1}\right|+\left|k_{2}\right|}{2}\right] .
$$

Thus positivity of curvature is sufficient for stability in this case. It is not necessary: we can have

$$
\left|k_{2}\right|<\frac{1}{4} B^{2}<\left|k_{1}\right|
$$

and still have

$$
\frac{\left|k_{1}\right|+\left|k_{2}\right|}{2}<\frac{1}{4} B^{2} .
$$

Since the arithmetic mean of positive numbers always exceed their geometric mean,

$$
\sqrt{k_{1} k_{2}}<\frac{\left|k_{1}\right|+\left|k_{2}\right|}{2}
$$

this would give stability without positivity of curvature.

On the other hand, negative curvature is sufficient for instability: $k_{3}<0$. 


\section{ACKNOWLEDGement}

I thank Sumit Das for explaining that the scalar field $\phi$ is the dilaton. In addition thanks to Miguel Alonso, Alex Iosevich, Andrew Jordan, Arnab Kar, Govind Krishnaswami and Evan Ranken for discussions.

\section{REFERENCES}

[1] M. P. do Carmo, Riemannian Geometry, Birkhauser (1992).

[2] S. S. Chern, Geometry without the Quadratic Restriction, Notices of the AMS, 959 (1996); D. Bao, R. L. Bryant, S. S. Chern and Z. Shen, A Sampler of Riemann-Finsler Geometry, Cambridge University Press (2004).

[3] G. Randers, Phys. Rev. 59, 195 (1941).

[4] W. R. Hamilton, Trans. Roy. Irish Acad., 17, 1-144 (1837).

[5] L. Klimes, Journal of Electromagnetic Waves and Applications, 27,1589(2013).

[6] A. Weinstein, Adv. Math. 6, 329 (1971).

[7] D. Bambusi "Birkhoff normal form and almost global existence for some Hamiltonian PDEs." (2007). Available at http://users.mat.unimi.it/users/bambusi/pedagogical.pdf

[8] R.J. Henderson and S.G. Rajeev, Class.Quant.Grav. 11, 1631 (1994), arXiv:gr-qc/9401029

[9] P.Bieliavsky, M.Cahen, S. Gutt and J. Rawnsley J. Geom. Phys. 38,140 (2001);P.Bieliavsky, M.Cahen, S. Gutt, J. Rawnsley and L. Schwachhofer, Symplectic Connections, arXiv:math/0511194 [math.SG]; K. Habermann and L. Habermann, Introduction to Symplectic Dirac Operators, Springer (2006)

[10] V. I. Arnold, Ann. Inst. Poly. Genoble 16 , 319 (1966)

[11] G. S. Krishnaswami and H. Senapati J. Math. Phys. 57, 102901 (2016), arXiv:1606.05091.

[12] R. Montgomery, A Tour of Subriemannian Geometries, Their Geodesics and Applications, AMS (2002)

[13] J. M. Lee, Riemannian Manifolds Springer (1997)

[14] Problem 3-10 in R. P. Feynman and A. R. Hibbs, Quantum Mechanics and Path Integrals, McGraw-Hill (1965)

[15] S. G. Rajeev, Advanced Mechanics, Oxford (2012).

Department of Physics and Astronomy, Department of Mathematics, University OF Rochester Rochester, NY 14627

E-mail address: s.g.rajeev@rochester.edu 